\title{
Is there a role for maintenance therapy after platinum chemotherapy in bladder cancer in the era of immune therapy?
}

\author{
Elie Rassy ${ }^{1}$, Tarek Assi*,1 $^{*}$ Joseph Kattan ${ }^{1}$ \\ ${ }^{1}$ Department of Oncology, Saint Joseph University, Faculty of Medicine, Beirut, Lebanon \\ *Author for correspondence: tarekassi@gmail.com
"The ICI maintenance strategy paradigm increases exposure to effective therapies especially through a switching approach in patients who achieve disease control after front-line chemotherapy"

First draft submitted: 15 September 2019; Accepted for publication: 4 October 2019; Published online: 15 November 2019

Keywords: bladder $\bullet$ immune checkpoint inhibitors $\bullet$ immunotherapy • maintenance $\bullet$ urothelial

Urothelial bladder carcinoma (UC) is the fourth most common cancer diagnosed among men and the second urological malignancy after prostate cancer $[1,2]$. Metastatic disease occurs in $50 \%$ of patients with muscle-invasive tumors despite systemic therapies and 10-15\% of patients at initial presentation [3]. The management of patients at the metastatic stage depends on a consensus statement of cisplatin-eligibility where the following criteria should be avoided: PS $>1$, stage III chronic kidney disease, grade $\geq 2$ audiometric loss, peripheral neuropathy and New York Heart Association class III heart failure [4]. Patients within this category are treated with cisplatin-containing combination chemotherapy, which has been the standard of care since the late 1980s demonstrating an overall survival (OS) of 12-14 months in different series [5]. Immune checkpoint inhibitors (ICI) have shown significant antitumor activity and durable responses in pretreated patients with metastatic UC [6,7]. Subsequently, ICI got approved in the front-line treatment of unfit patients with PDL-1 positive expression and in the second-line treatment of patients pretreated with a platinum-based chemotherapy. Maintenance therapy after clinical benefit from platinum-based therapy was recently evaluated by switch chemotherapy or targeted therapy or even ICI. The main goal of this strategy is to reduce or postpone disease relapse in patients who benefited from first-line chemotherapy with prevention of symptoms worsening by using effective and safe drugs. Maintenance therapy demonstrated promising outcomes in certain types of solid tumors such as ovarian, lung and colorectal cancer but is not yet considered a standard of care in UCs [8-11]. In this paper, we opted to evaluate the role of maintenance strategy in metastatic urothelial carcinoma (mUC) in order to understand the optimal sequence of treatment administration.

\section{Switch maintenance}

The maintenance therapy with conventional and targeted agents did not significantly impact the oncological outcomes of patients with metastatic UC [12]. Three prospective randomized controlled trials have been published in this regard. The first trial is a Phase II multicentric study (MAJA; SOGUG 2011/02) that randomized 88 patients with mUC who had disease control after 4-6 cycles of platinum-based chemotherapy with gemcitabine to receive vinflunine (280-320 mg/sqm every 3 weeks; 45 pts) or best supportive care ( 43 pts). Vinflunine, a vinca-alcaloid antineoplastic agent that targets microtubules, is approved by the European Medicines agency in the relapsed setting of mUC after demonstrating significant improvement in progression-free survival (PFS), overall response rate but not in OS in the intention-to treat population [13]. The experimental arm demonstrated PFS (6.2 vs 4 months $[\mathrm{HR}=0.59,95 \% \mathrm{CI}: 0.37-0.96])$ and OS (16.7 vs 13.2 months) benefit. The overall response rate was also in favor of the experimental arm (21 vs 7\%) but with more common gr. 3-4 side effects with vinflunine neutropenia (18\% vs 0 ), fatigue ( 16 vs $2 \%$ ) and constipation ( $14 \%$ vs 0$)$. In a post-hoc multivariate analysis including all stratification

Future 8 Medicine 
factors, only vinflunine therapy and liver metastasis were associated with prolonged PFS [14]. The second trial used the same study design and randomized patients with advanced mUC who had disease control after 4-6 cycles of chemotherapy to maintenance therapy with sunitinib (50 mg dose daily for 28 days on a cycle of 6 weeks) (26 pts), an anti-angiogenic tyrosine kinase inhibitor or placebo (28 pts). The median PFS (2.9 vs 2.7 months, $[\mathrm{HR}=1,95 \% \mathrm{CI}: 0.6-1.8]$ ) and OS [10.5 vs 10.3 months]) were not statistically different between the two arms but the study was terminated earlier due to slow accrual [15]. Grade 3-4 adverse events in the sunitinib group were thrombocytopenia (30.8\%), fatigue (19.2\%), diarrhea and mucositis (15.4\%) and hypertension (11.4\%) The third is a Phase III trial that enrolled patients with HER1-HER2 positive (3+ on immunohistochemistry) mUC who had disease control after 4-8 cycles of platinum-based chemotherapy to receive lapatinib (1500 mg daily; 116 pts) or placebo (116 pts). The median PFS (4.5 vs 5.1 months, [HR $=1.07,95 \%$ CI: $0.81-1.43]$ ) and OS (12.6 vs 12 months, [HR $=0.96,95 \%$ CI: 0.70-1.31]) were not statistically different in the two arms [16].

The preclinical scientific rationale for ICI maintenance following chemotherapy is intriguing although not clearly documented. Carcinogenesis occurs in an evolving immune environment where the immune system initially eliminate tumors cells who develop immunosuppressive properties leading to immune escape and ultimately disease progression [17]. Chemotherapy exerts immunomodulatory changes that impacts regulatory $\mathrm{T}$ cells and myeloidderived suppressor cell. The effects of chemotherapy vary with the specific agents and doses administered [18]. The relevant drugs in bladder cancer namely gemcitabine and platinum agents are immunosuppressive thus can deplete regulatory $T$ cells and increase T-cell proliferation and natural killer cell lytic activity [19,20]. PD-L1-targeted approaches can overcome immune-evasion mechanisms elicited by chemotherapy and augment immune-mediated tumor control.

In this regard, the Phase II double-blind randomized controlled trial (NCT02500121) has assessed the role of ICI maintenance in mUC [21]. The study randomized 107 patients with metastatic UC who achieve stable disease following $\leq 8$ cycles of platinum-based regimen to maintenance pembrolizumab (200 $\mathrm{mg}$ intravenously [iv.] every 3 weeks; 55 pts) or placebo ( 52 pts) for a total of 24 months followed by observation until recurrence. The crossover to pembrolizumab in case of disease progression under placebo was permitted. The pembrolizumab arm achieved longer PFS (5.4 vs 3.2 months, [HR $=0.64,95 \%$ CI: $0.41-0.98]$ ) and 18 -month restricted mean PFS (8.1 vs 5.5 months; $\mathrm{p}=0.023)$. It also showed higher overall response (22 vs $12 \%)$ and disease control rate (57 and 41\%). Overall, grade 3-4 adverse events were reported in 53\% with ICI and 35\% with placebo. The most common side effects reported being fatigue ( 7 vs $0 \%$ ), increased liver enzymes ( 5 vs $0 \%$ ) and dyspnea ( 5 vs 0\%) [21]. Another ongoing trial in this regard is the JAVELIN Bladder 100 study (NCT02603432) is evaluating maintenance avelumab in patients with advanced UC who have completed at least four cycles of platinum-based chemotherapy without evidence of disease progression.

\section{Continuous maintenance}

With the introduction of ICIs at earlier stages in the management of mUC, the role of maintenance therapy has been put into question. Recently, an announcement has confirmed that the addition of atezolizumab to platinumbased regimen in the first-line setting of LA- or mUC-improved PFS in the Phase III trial (IMvigor130). In this trial, patients with LA or mUC were randomized to receive atezolizumab (1200 mg iv. every 3 weeks) alone, in combination to chemotherapy (gemcitabine $1000 \mathrm{mg} / \mathrm{sqm} \mathrm{d} 1$ and $\mathrm{d} 8$ with carboplatin area under the curve 4.5 $\mathrm{d} 1$ or Cisplatin $70 \mathrm{mg} / \mathrm{sqm} \mathrm{d} 1$ every 3 weeks) or chemotherapy alone [22]. With the addition of ICIs at earlier stages of the disease, the role of switch-maintenance is questionable, whereas future trials should assess the role of continuous maintenance ICIs in those exhibiting response after first-line immunotherapy.

\section{Conclusion}

The ICI maintenance strategy paradigm increases exposure to effective therapies especially through a switching approach in patients who achieve disease control after front-line chemotherapy. Usually, a high percentage of patients progressing after the first line of chemotherapy never receive second-line therapy at the time of progression. The maintenance strategy ensures that the majority of patients receive ICI, which have proven efficacy and durable response in mUC. The available biological and clinical data support this hypothesis and required further validation in Phase III trials. Questions concerning the efficacy of this strategy remain unanswered. 


\section{Financial \& competing interests disclosure}

The authors have no relevant affiliations or financial involvement with any organization or entity with a financial interest in or financial conflict with the subject matter or materials discussed in the manuscript. This includes employment, consultancies, honoraria, stock ownership or options, expert testimony, grants or patents received or pending, or royalties.

No writing assistance was utilized in the production of this manuscript.

\section{References}

1. Malats N, Real FX. Epidemiology of bladder cancer. Hematolo. Oncol. Clin. North Am. 29(2), 177-189 (2015).

2. Siegel RL, Miller KD, Jemal A. Cancer statistics, 2019. CA A Cancer J. Clin. 69(1), 7-34 (2019).

3. Rosenberg JE, Carroll PR, Small EJ. Update on chemotherapy for advanced bladder cancer. J. Urol. 174(1), 14-20 (2005).

4. Galsky MD, Hahn NM, Rosenberg J et al. Treatment of patients with metastatic urothelial cancer "unfit" for cisplatin-based chemotherapy. J. Clin. Oncol. 29(17), 2432-2438 (2011).

5. Bellmunt J, Petrylak DP. New therapeutic challenges in advanced bladder cancer. Semin. Oncol. 39 (5),598-607 (2012).

6. Aoun F, Rassy EE, Assi T, Albisinni S, Katan J. Advances in urothelial bladder cancer immunotherapy, dawn of a new age of treatment. Immunotherapy 9(5), 451-460 (2017).

7. Rassy EE, Bakouny Z, Aoun F et al. A network meta-analysis of the PD(L)-1 inhibitors in the salvage treatment of urothelial bladder cancer. Immunotherapy 10(8), 657-663 (2018).

8. Paz-Ares L, de Marinis F, Dediu M et al. Maintenance therapy with pemetrexed plus best supportive care versus placebo plus best supportive care after induction therapy with pemetrexed plus cisplatin for advanced non-squamous non-small-cell lung cancer (PARAMOUNT): a double-blind, Phase III, randomised controlled trial. Lancet Oncol. 13(3), 247-255 (2012).

9. Mirza MR, Monk BJ, Herrstedt J et al. Niraparib maintenance therapy in platinum-sensitive, recurrent ovarian cancer. N. Eng. J. Med. 375(22), 2154-2164 (2016).

10. Ledermann J, Harter P, Gourley C et al. Olaparib maintenance therapy in platinum-sensitive relapsed ovarian cancer. $N$. Eng. J. Med. 366(15), 1382-1392 (2012).

11. Giuliani F, De Vita F, Colucci G, Pisconti S. Maintenance therapy in colon cancer. Cancer Treat. Rev. 36, S42-S45 (2010)

12. Hoffman-Censits J, Wong Y-N. Perioperative and maintenance therapy after first-line therapy as paradigms for drug discovery in urothelial carcinoma. Clin. Genitourin. Cancer 13(4), 302-308 (2015).

13. Bellmunt J, Théodore C, Demkov T et al. Phase III trial of vinflunine plus best supportive care compared with best supportive care alone after a platinum-containing regimen in patients with advanced transitional cell carcinoma of the urothelial tract. J. Clin. Oncol. 27(27), 4454-4461 (2009).

14. García-Donas J, Font A, Pérez-Valderrama B et al. Maintenance therapy with vinflunine plus best supportive care versus best supportive care alone in patients with advanced urothelial carcinoma with a response after first-line chemotherapy (MAJA; SOGUG 2011/02): a multicentre, randomised, controlled, open-label, Phase II trial. Lancet Oncol. 18(5), 672-681 (2017).

15. Grivas PD, Daignault S, Tagawa ST et al. Double-blind, randomized, Phase II trial of maintenance sunitinib versus placebo after response to chemotherapy in patients with advanced urothelial carcinoma. Cancer 120(5), 692-701 (2014).

16. Powles T, Huddart RA, Elliott T et al. Phase III, double-blind, randomized trial that compared maintenance lapatinib versus placebo after first-line chemotherapy in patients with human epidermal growth factor receptor 1/2-positive metastatic bladder cancer. J. Clin. Oncol. 35(1), 48-55 (2017).

17. Swann JB, Smyth MJ. Immune surveillance of tumors. J. Clin. Invest. 117(5), 1137-1146 (2007).

18. Kersten K, Salvagno C, de Visser KE. Exploiting the immunomodulatory properties of chemotherapeutic drugs to improve the success of cancer immunotherapy. Front. Immunol. 6, 516 (2015).

19. Shevchenko I, Karakhanova S, Soltek S et al. Low-dose gemcitabine depletes regulatory T cells and improves survival in the orthotopic Panc02 model of pancreatic cancer. Int. J. Cancer 133(1), 98-107 (2013).

20. de Biasi AR, Villena-Vargas J, Adusumilli PS. Cisplatin-induced antitumor immunomodulation: a review of preclinical and clinical evidence. Clin. Cancer Res. 20(21), 5384-5391 (2014).

21. Galsky MD, Pal SK, Mortazavi A et al. Randomized double-blind Phase II study of maintenance pembrolizumab versus placebo after first-line chemotherapy in patients (pts) with metastatic urothelial cancer (mUC): HCRN GU14-182. Am. Soc. Clin. Oncol. 37(15), 4504 (2019).

22. Galsky MD, Grande E, Davis ID et al. IMvigor130: a randomized, phase III study evaluating first-line (1L) atezolizumab (atezo) as monotherapy and in combination with platinum-based chemotherapy (chemo) in patients (pts) with locally advanced or metastatic urothelial carcinoma (mUC). Am. Soc. Clin. Oncol. 36, Suppl. 15 (2018). 
